\title{
Carbon Nanotube Mechanical Resonators for Mass Sensing
}

\author{
Seiji Akita ${ }^{1,2, *}$ and Takayuki Arie ${ }^{1,2}$ \\ 'Department of Physics and Electronics, Graduate School of Engineering, Osaka Prefecture University, \\ 1-1 Gakuen-cho, Naka-ku, Sakai, Osaka 599-8531, Japan \\ ${ }^{2}$ CREST, Japan Science and Technology Agency (JST), Kawaguchi Center Building 4-1-8, \\ Honcho, Kawaguchi-shi, Saitama 332-0012, Japan
}

(Received March 5, 2009; accepted June 1, 2009)

Key words: carbon nanotube, mechanical resonator, nano-electro-mechanical system, mass sensing, cantilever

We demonstrated the application of a mechanical resonantor of a nanotube cantilever to minute mass detection in vacuum and at atmospheric pressure. For a vacuum environment, a method for measuring the oscillation amplitude of a nanotube cantilever using an electron beam of a scanning electron microscope was proposed. A quality factor of $\sim 1,000$ for the nanotube resonator in vacuum was revealed and a resolution of the resonant frequency of $\sim 10 \mathrm{~Hz}$ was achieved, which corresponds to a mass range of less than $100 \mathrm{zg}$ at room temperature in vacuum. We also performed in situ measurements of densities of electron-beam-induced amorphous carbon with sizes of less than $100 \mathrm{~nm}$. Furthermore, an optical detection method for detecting the vibration of nanotube resonators in air was also proposed. The motion of the nanotube resonator was detected by the fluctuation of scattered light. A higher sensitivity for the detection of the vibration was achieved by using the interference induced by multiple scattered lights. We demonstrated that a quality factor of $\sim 40$ for the nanotube resonator corresponds to a resolution of a mass range of approximately $1 \mathrm{ag}$ at room temperature in air.

\section{Introduction}

Force sensing using a resonant-frequency shift of a cantilevered resonator is expected to provide a high sensitivity. Cantilever miniaturization is crucial in bringing highly sensitive detection into practice. Therefore, nanoscale mechanical resonators are widely studied as highly sensitive mass sensors. To improve sensitivity, the high quality factor $Q$ of oscillators is advantageous for highly sensitive mass sensing using nanoscale resonators. $^{(1-4)}$ It is noted that the $Q$ factor is directly correlated to the energy loss of a vibrating system. Carbon nanotubes are appropriate for mass sensor application ${ }^{(5-9)}$ because of their light weight, high aspect ratio, and extraordinary mechanical properties. ${ }^{(10-12)}$

"Corresponding author: e-mail: akita@pe.osakafu-u.ac.jp 
For the mass sensor application of nanotubes, we must detect the mechanical motion of nanotubes. The electron beam in an electron microscope has been used to detect the mechanical motion of nanotube resonators. ${ }^{(5-8)}$ The field-emission current from the cantilevered nanotube was also used as the probe of motion detection. ${ }^{(9)}$ Recently, the atomic mass resolution has been realized by using this detection method. ${ }^{(9)}$

These methods are applicable only in vacuum, while conditions in air or in liquid are required for measuring the mass of biomolecules. In a viscous fluid, such as air or liquid, the strong damping of vibration by the fluid affects the $Q$ factor. Individual nanotubes should be characterized to ascertain their capability for the application of high-performance mass sensors in viscous fluids. To overcome this problem, other techniques for detecting the nanotube motion, such as an optical detection method, are required. However, a conventional optical detection method for microscale cantilevers is difficult to apply to nanotubes because of their size limitation. In contrast, the scattered light from small objects with sizes less than optical diffraction limits can be detected and the motion of the objects can be detected by the fluctuation of the scattered light.

In this study, we demonstrated the application of a nanotube resonator to minute mass detection in vacuum and at atmospheric pressure.

\section{Preparation of Cantilevered Nanotube Resonators}

Two types of multiwalled nanotube were examined in this study; one was synthesized by a well-controlled arc discharge method, ${ }^{(13)}$ by which the nanotubes are highly crystallized, and the other, which is commercially available, was synthesized by chemical vapor deposition (CVD) with postannealing treatment at temperatures higher than $1,500^{\circ} \mathrm{C}$. The G/D ratio of the Raman spectrum after the postannealing treatment is greater than 10. Therefore, the nanotubes synthesized by CVD after the postannealing treatment have a highly graphitized structure and a less amorphous carbon layer. The nanotubes were aligned at a Pt-coated knife edge by an $\mathrm{AC}$ electrophoresis technique to produce cantilevered nanotube arrays. ${ }^{(14)}$

\section{Vibration Detection of Nanotube Resonators in Scanning Electron Microscope $^{(6)}$}

A nanotube array was set on a stage with a piezoelectric device in a scanning electron microscope and then oscillated mechanically by applying an AC voltage to the piezoelectric device. The tip of one nanotube cantilever was irradiated using an electron beam with a diameter smaller than $1 \mathrm{~nm}$ in a spot mode; it was used for probing the resonant frequency and oscillation amplitude of the nanotube resonator. ${ }^{(6,7)}$ For simplicity, only the harmonic oscillation of the first mode was assumed for the vibration of the nanotube cantilever. In this vibration mode, the resonant frequency $f$ is related to $^{(15)}$

$$
f=\frac{1.875^{2}}{2 \sqrt{3} \pi} \sqrt{\frac{3 Y I}{m_{0} L^{3}}}
$$


where $I$ is the moment of inertia given by $\pi\left(r_{\mathrm{o}}^{4}-r_{\mathrm{i}}^{4}\right) / 4, Y$ Young's modulus, $m_{0}$ the mass of the nanotube, and $r_{\mathrm{o}}$ and $r_{\mathrm{i}}$ the outer and inner radii of the nanotube, respectively. From the resonant frequency, we can obtain the Young's modulus of each nanotube using eq. (1). The $Q$ factors for several arc-produced nanotubes examined in this experiment were varied in the range of 300-2,000 with a mean of $\sim 1,000$. The $Q$ factors and Young's moduli for the CVD-synthesized nanotubes are respectively lower than 200 and 0.3 $\mathrm{TPa}$, which are smaller than those for the arc-produced nanotubes.

The electron beam for the probe induced the deposition of amorphous carbon (a-C) on the nanotube. Figure 1 shows scanning electron microscopy (SEM) images of the nanotube oscillation and a-C particulate deposited on a nanotube tip. The mass of the deposited particle shown in Fig. 1 increased with time, which was measured as a shift of the resonant frequency $f$. It is reasonable to infer from the figure that the particle is spherical. The particle radius is $R=80 \mathrm{~nm}$ for an exposure time of $400 \mathrm{~s}$. It is also reasonable to consider that $R^{3}$, i.e., the mass $m\left(=4 \pi R^{3} \rho / 3\right)$ of the particle is proportional to the exposure time, where $\rho$ represents the particle density.

For the first-order harmonic oscillation, $f$ is approximately related to $m$ according to $^{(15)}$

$$
f=\frac{1.875^{2}}{2 \sqrt{3} \pi} \sqrt{\frac{k}{m_{0}+4 m}},
$$

where $k$ is the spring constant of the nanotube cantilever that is given by $3 Y I / L^{3}$. From eq. (2), we have

$$
R^{3}=\frac{3}{16 \pi \rho}\left(\frac{1.875^{4}}{12 \pi^{2}} \frac{k}{f^{2}}-m_{0}\right)
$$

Figure 2 shows $R^{3}$ plotted as a function of $1 / f^{2}$. It is evident that $R^{3}$ is proportional to $1 / f^{2}$, which agrees well with eq. (3). This fact indicates that the nanotube behaves as

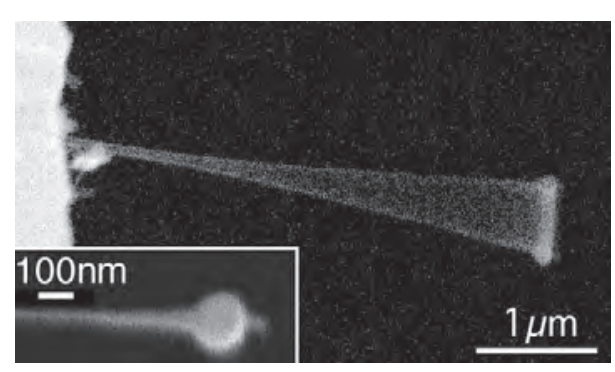

Fig. 1. SEM image of a carbon nanotube oscillator. The inset shows the deposit generated by an electron beam deposition process. 


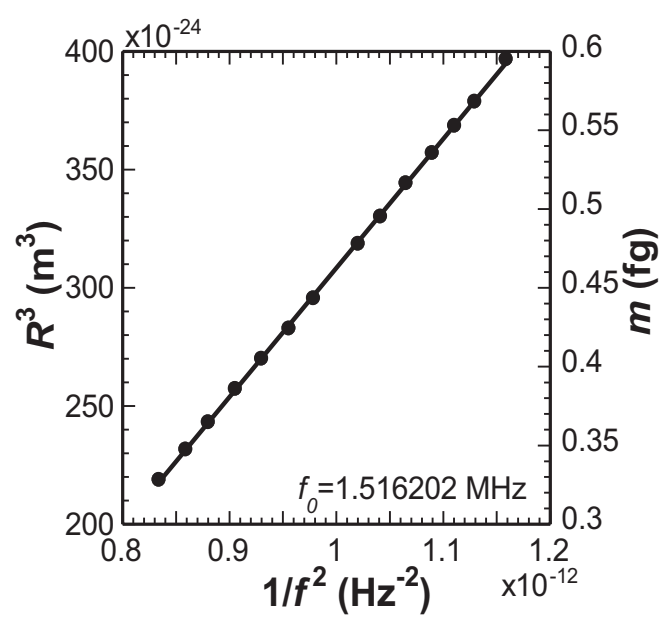

Fig. 2. Resonant frequency dependences of the radius and mass of the deposit.

an ideal resonator. $\rho$ is estimated to be $0.36 \mathrm{~g} / \mathrm{cm}^{3}$ from the y-intercept, where $m_{0}=1.41$ fg is obtained from the parameters $r_{\mathrm{o}}=7.5 \mathrm{~nm}$ and $r_{\mathrm{i}}=1 \mathrm{~nm}$ measured by transmission electron microscopy (TEM), and $L=3.6 \mu \mathrm{m}$ and $\rho_{0}=2.25 \mathrm{~g} / \mathrm{cm}^{3}$ (density of graphite). The estimated $\rho$ for a-C is much smaller than that obtained by a focused ion beam (FIB) process $\left(\sim 2 \mathrm{~g} / \mathrm{cm}^{3}\right){ }^{(16)}$ In the present stage, we speculate that the a-C fabricated by the electron beam with an acceleration voltage of $5 \mathrm{kV}$ has a porous structure. This is a subject for further study. From the x-intercept, $f_{0}$ (free oscillation frequency of the nanotube) and $Y$ are also estimated to be $1.5162 \mathrm{MHz}$ and $0.23 \mathrm{TPa}$, respectively. In this manner, we can easily investigate the density of the deposit induced by electron beam irradiation. This ease of investigation is useful for evaluating the quality of deposits induced by the electron and focused ion beams, which are widely used for nanostructure fabrication.

The mass of the deposited particle obtained from $\rho$ is also shown in Fig. 2. The measured mass is less than $10^{-15} \mathrm{~g}$ and resolved in a range of $10^{-18} \mathrm{~g}$. By considering the minimum detectable mass based on the thermal noise, ${ }^{(17)}$ the sensitivity of mass detection is $c a .100 \mathrm{zg}$ (zeptogram, $10^{-21} \mathrm{~g}$ ) for the nanotube shown in Fig. 1 at room temperature. Shorter nanotubes can be expected to detect a zeptogram or less, corresponding to the mass of a single molecule.

\section{Optical Detection of Nanotube Resonators in Air}

The CVD-grown nanotube cantilever array was set on a piezoelectric oscillator in a chamber for optical microscopy and then oscillated mechanically by applying an AC voltage to the piezoelectric oscillator in air. The nanotube cantilever array was irradiated using a laser beam with a wavelength of $532 \mathrm{~nm}$ under the conditions of a dark-field illumination mode. The scattered light from a certain nanotube cantilever was collected by an objective lens $(\times 50, \mathrm{NA}=0.7)$ and transported to a $\mathrm{CCD}$ camera, as schematically 
shown in Fig. 3. Individually protruding nanotubes are clearly observed in the image shown in Fig. 4. In this image, the shortest protrusion of the nanotube is $2 \mu \mathrm{m}$ in length. Furthermore, some fringe patterns induced by the scattered light are also observed.

Figure 5(a) shows a SEM image of one of the nanotube cantilevers used in this experiment, the length and diameter of which are $13 \mu \mathrm{m}$ and $75 \mathrm{~nm}$, respectively. Figure 5 (b) shows an optical microscopy image of a nanotube cantilever. Figure 5(c) shows an intensity profile across the nanotube cantilever. The full width at half maximum (FWHM) of the nanotube cantilever is $\sim 1 \mu \mathrm{m}$, which corresponds to the width of $50 \mu \mathrm{m}$ estimated from the magnification of the objective lens at the focal plane on the CCD. Since the

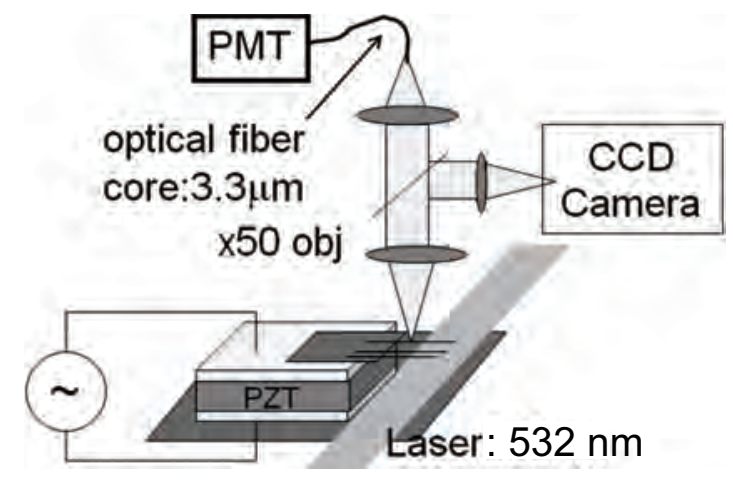

Fig. 3. Schematic diagram of the experimental setup for optical detection.

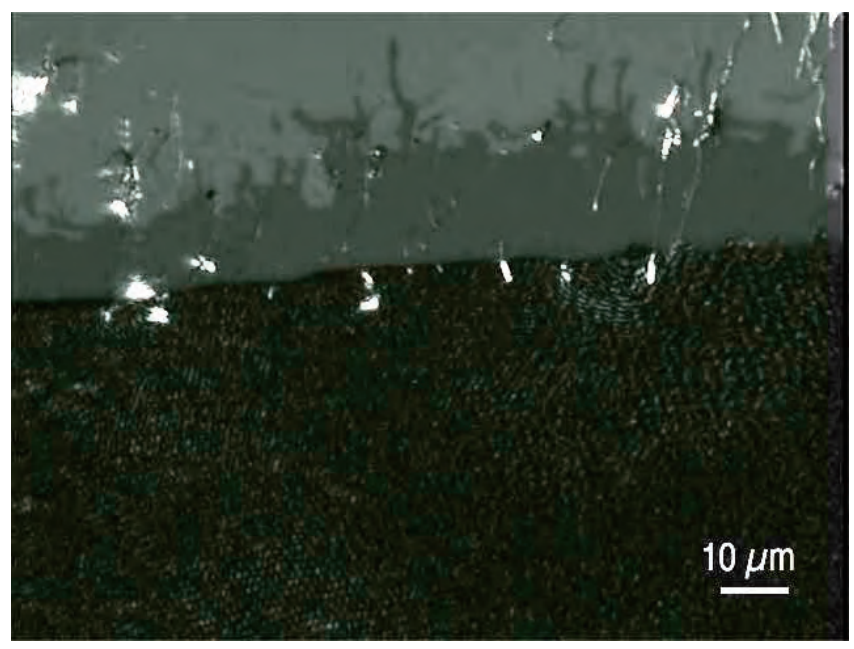

Fig. 4. Dark-field optical microscopy image of nanotube cantilever array: a laser with a wavelength of $532 \mathrm{~nm}$ was used as the light source. 

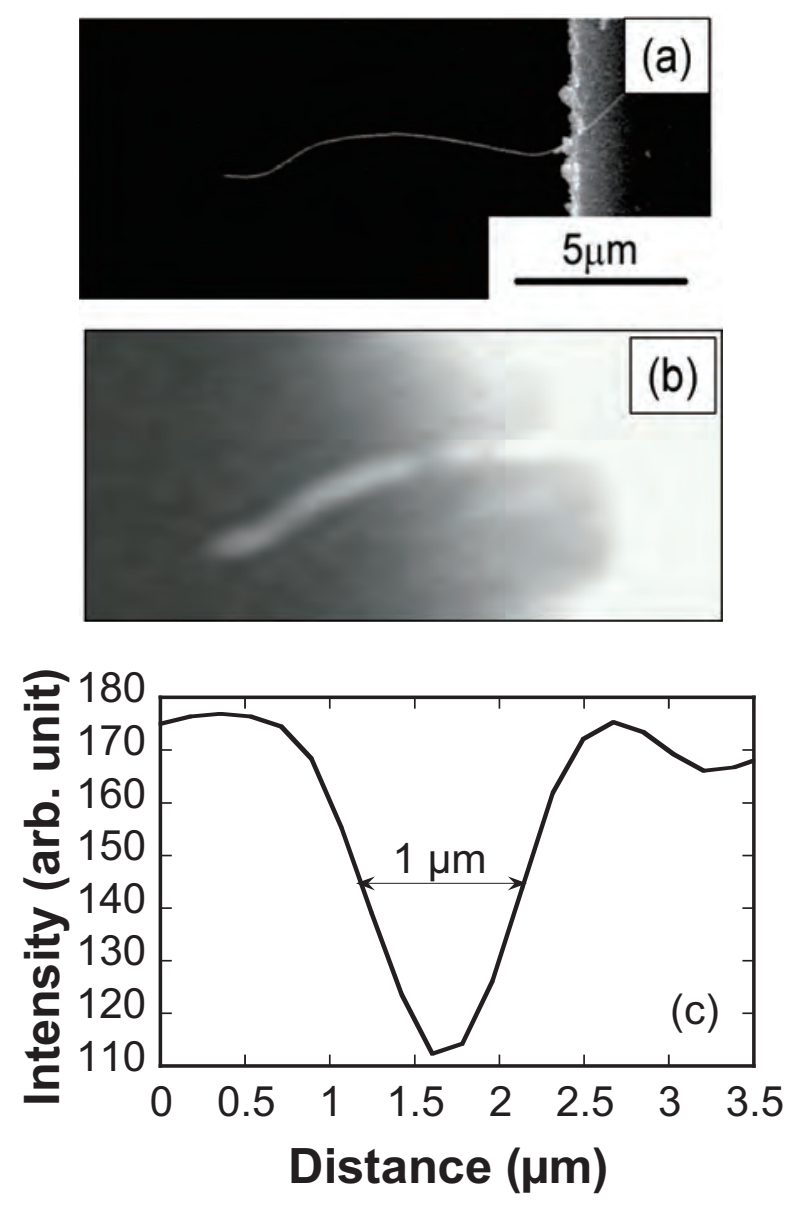

Fig. 5. Typical nanotube cantilever: (a) SEM image, (b) optical microscopy image in off-resonant state, and (c) intensity profile across the nanotube cantilever shown in (b).

effective pixel size of the CCD camera is $\sim 10 \mu \mathrm{m}$, the fluctuation of the scattered light from the nanotube is sufficiently detected with a sub-micrometer order.

Figures 6(a) and 6(b) show a nanotube cantilever in the off-resonant and resonant states in air, respectively. The width of the tip of the nanotube in the resonant state is slightly larger than that in the off-resonant state. Thus, we have succeeded in the optical detection of the vibrating nanotubes in air. To extract the frequency dependence of vibrating amplitude from the image, an image processing procedure was performed for each excitation frequency as follows. The square of the difference in pixel brightness between the off-resonant state and the vibrating image at a certain excitation frequency was obtained for a certain area in the optical images, which corresponds to the difference in area between the areas for the vibrating and off-resonant nanotubes. The difference in area is roughly proportional to the product of the length and vibrating amplitude of the 


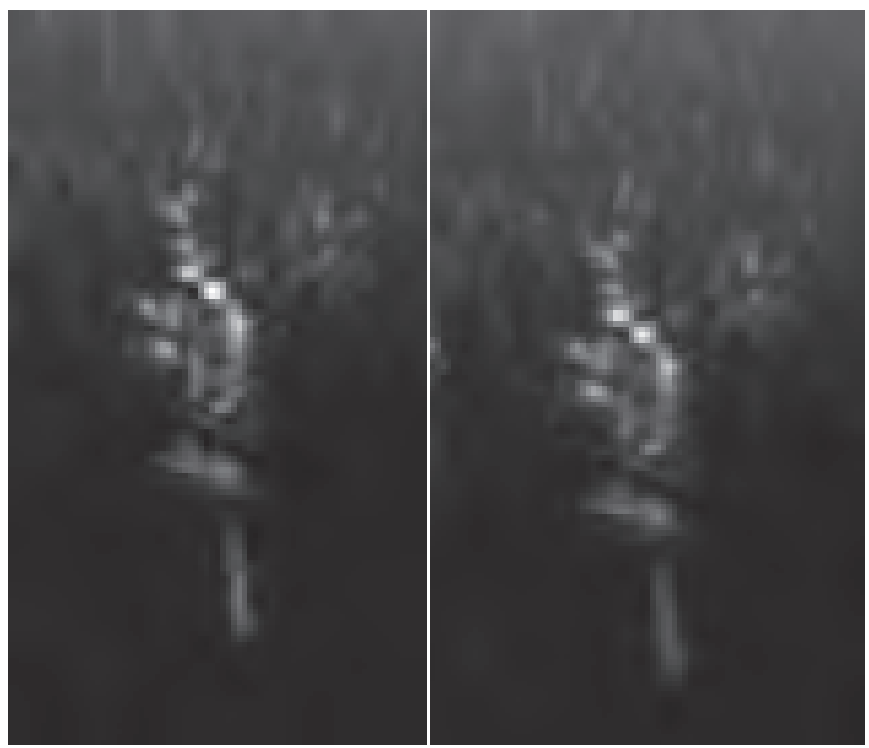

(a)

(b)

Fig. 6. Optical microscopy images of a nanotube cantilever in (a) off-resonant and (b) resonant states in air.

nanotube. As a result, the square root of the sum is roughly proportional to the vibrating amplitude for each excitation frequency at a small vibrating amplitude.

Figure 7 shows the resonant curve of the nanotube resonator shown in Fig. 6 obtained by the previous procedure. The resonant frequency and $Q$ factor of the nanotube resonator are $985 \mathrm{KHz}$ and 40, respectively. From the thermal noise analysis of mechanical resonators, the theoretical limit of mass sensing using the nanotube resonator becomes sub-attogram, as estimated from the resonant frequency and $Q$ factor. Thus, the nanotube resonator acts as a highly sensitive mass sensor even in air. It should be noted that the shorter and thinner nanotube shows a higher sensitivity because of lighter resonators.

Although the optical detection of the vibration of the nanotube could be achieved by simple dark-field observation, a higher sensitivity for displacement detection is required to measure the shorter and thinner nanotube. Note that we used laser light for the irradiation of the nanotube. The scattered lights from the nanotube and other portions interfere with each other, as schematically shown in Fig. 8. These scattered lights produce a fringe pattern in the optical image because of the coherence of the laser light. In this case, if only the nanotube slightly moves, the fringe pattern will be changed. 


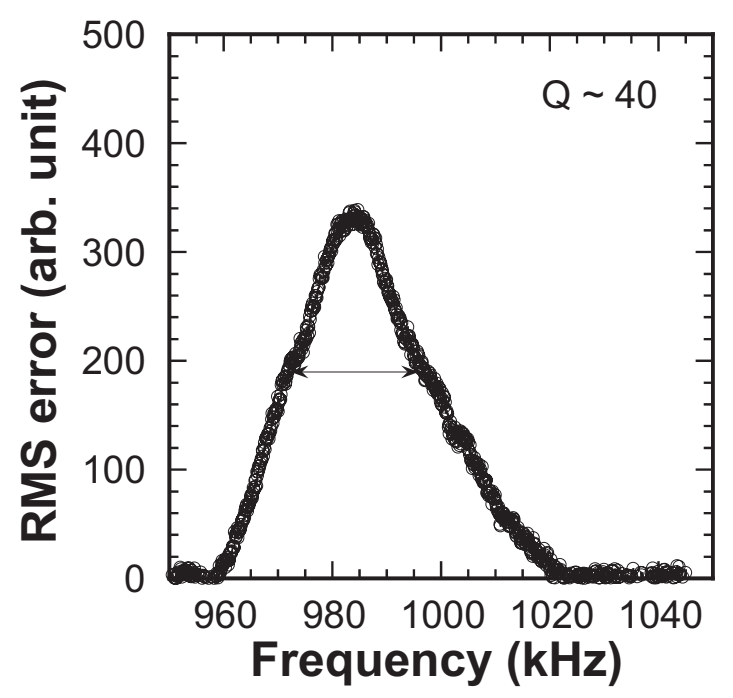

Fig. 7. Resonant curve of the nanotube resonator shown in Fig. 6.

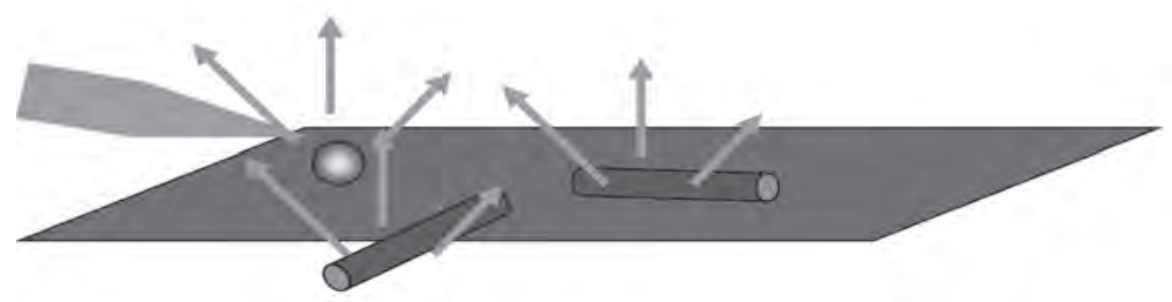

Fig. 8. Schematic illustration of interference pattern formation by multiple scattered light.

We expect that this interferometric effect will be useful for the detection of the small displacement of a nanomaterial.

To examine the effectiveness of this interferometric method, we have calculated the difference between the scattered light intensities before and after the $50 \mathrm{~nm}$ movement of the nanomaterial using the 2-dimentional finite element method (2D-FEM). Figure 9(a) shows a model for the calculation, where two nanotubes with a diameter of $20 \mathrm{~nm}$ were set in parallel with a $1.41 \mu \mathrm{m}$ separation and perpendicular to the image plane, where the nanotubes were assumed to be a perfect electric conductor for simplicity. The light with a wavelength of $500 \mathrm{~nm}$ was allowed to propagate in the $x$-direction in a TE 


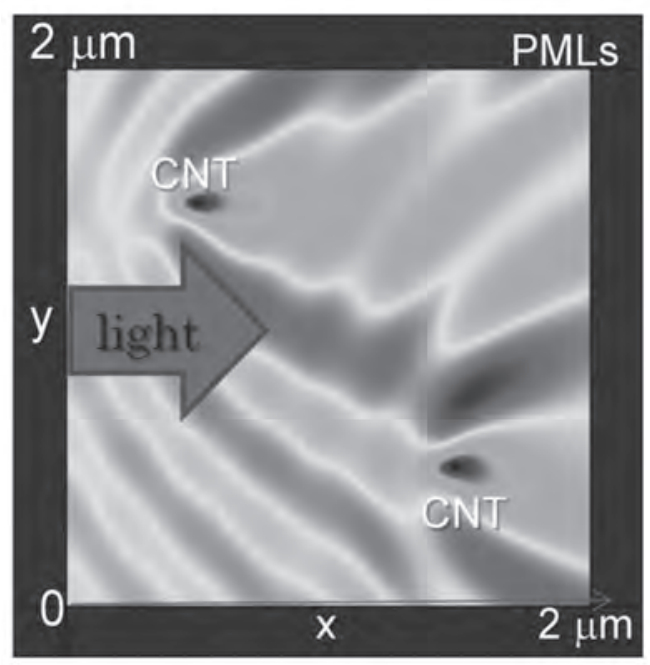

(a)

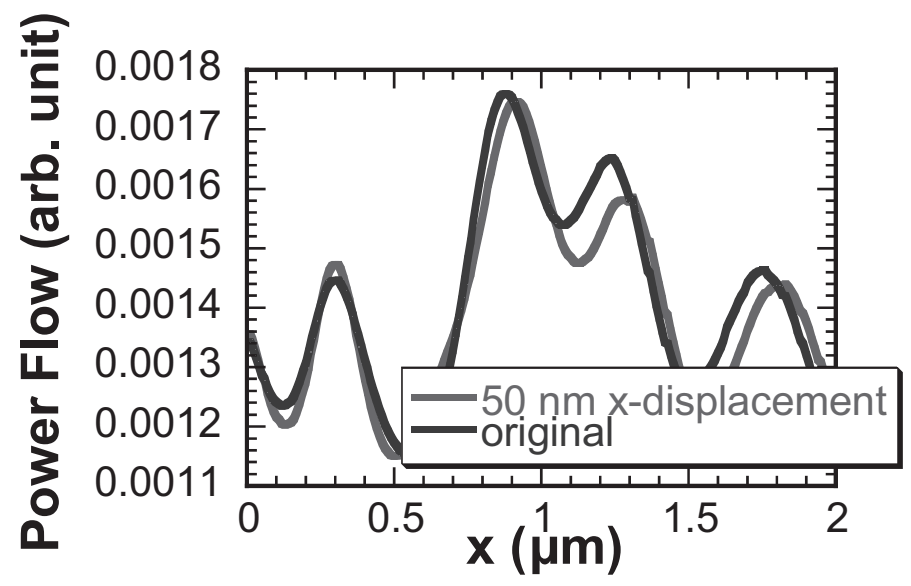

(b)

Fig. 9. 2D-FEM calculation of the interference of scattered lights from two nanotubes: (a) model for calculation and (b) the intensity profiles along the $x$-direction at $y=2 \mu \mathrm{m}$ before and after the $50 \mathrm{~nm}$ displacement of one of the nanotubes.

mode. Perfectly matched layers (PMLs) with an additional domain that absorbs the incident radiation without producing reflections were set around the model to prevent the multireflection of light at the boundary. The light was scattered by the two nanotubes and the scattered lights induced the interference. The interfered light intensities at the plane of $y=2 \mu \mathrm{m}$ before and after the displacement are shown in Fig. 9(b). The interference intensity around $x=1.2 \mu \mathrm{m}$ was changed by $5 \%$ by the displacement. Thus, 


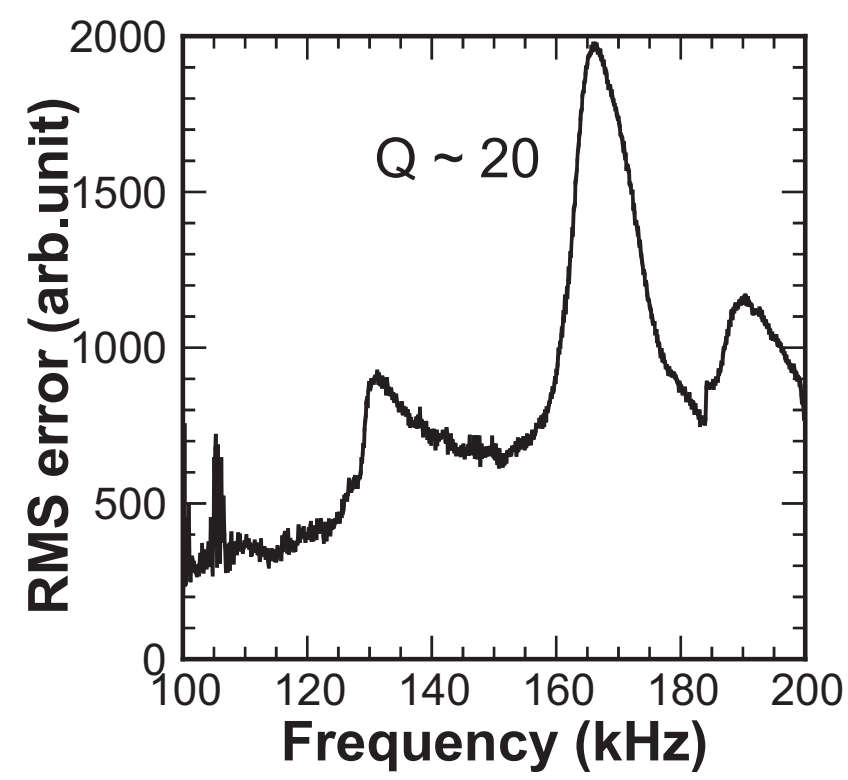

Fig. 10. Frequency response curve extracted from the interferometric pattern change.

the interferometric method is effective for detecting the nanomaterial displacement in the nm range.

This interferometric method was applied to the nanotube resonator. To obtain the interferometric image, we have slightly changed the focal plane of the microscope from the just-in-focus position. This defocusing position corresponds to the $y=2 \mu \mathrm{m}$ plane described in the FEM calculation. The interferometric pattern is clearly observed, as shown in this image. The frequency response curve extracted from the interferometric pattern change is shown in Fig. 10. Note that no signals of the displacement around this frequency range could be detected from the simple dark field image. Thus, we can successfully measure the frequency response curve for the nanotube resonator using the interferometric method with a high sensitivity. We believe that this method enables the detection of the displacement of the nanotube resonator in a highly viscous fluid, such as water.

\section{Conclusions}

We demonstrated the application of a nanotube cantilever to minute mass detection in vacuum and at atmospheric pressure. In a vacuum environment, the oscillation amplitude of a nanotube cantilever was measured by using an electron beam. A quality factor of $\sim 1,000$ for the nanotube cantilever in vacuum was revealed and the resolution 
of the resonant frequency was determined to be $\sim 10 \mathrm{~Hz}$, which corresponds to a mass range less than $100 \mathrm{zg}$ at room temperature in vacuum.

We also performed in situ measurements of densities of electron-beam-induced amorphous carbon with sizes of less than $100 \mathrm{~nm}$. In addition, we investigated an optical method for detecting the vibration of an individual nanotube in air. The vibration amplitude of the cantilevered CVD-synthesized nanotube was successfully measured using a dark-field optical microscopy image. The $Q$ factor in air was measured to be $\sim 40$, which is lower than that measured in vacuum. This is due to the collision of the nanotube with the molecules in air. We considered that the interferometric method based on the scattering from the nanomaterial is effective for detecting the displacement of a nanomaterial. The expected mass resolution at atmospheric pressure becomes approximately $1 \mathrm{ag}$ at room temperature. Thus, the nanotube resonators are very efficient for measuring the minute mass even in a viscous fluid.

\section{Acknowledgements}

This work was supported in part by a Grant-in-Aid for Scientific Research from the Japan Society for the Promotion of Science.

\section{References}

1 F. R. Blom, S. Bouwstra, M. Elwenspoek and J. H. Fluitman: J. Vac. Sci. Technol. B 10 (1992) 19.

2 J. Yang, T. Ono and M. Esashi: Appl. Phys. Lett. 77 (2000) 3860.

3 T. Ono, X. Li, H. Miyashita and M. Esashi: Rev. Sci. Instrum. 74 (2003) 1240.

4 S. S. Verbridge, J. M. Parpia, R. B. Reichenbach, L. M. Bellan and H. G. Craighead: J. Appl. Phys. 99 (2006) 124304.

5 P. Poncharal, Z. L. Wang, D. Ugarte and W. A. de Heer: Science 283 (1999) 1513.

6 M. Nishio, S. Sawaya, S. Akita and Y. Nakayama: Appl. Phys. Lett. 86 (2005) 133111.

7 M. Nishio, S. Sawaya, S. Akita and Y. Nakayama: J. Vac. Sci. Technol. B 23 (2005) 1975.

8 S. Sawaya, S. Akita and Y. Nakayama: Appl. Phys. Lett. 89 (2006) 193115.

9 K. Jensen, K. Kim and A. Zettl: Nat. Nanotechnol. 3 (2008) 533.

10 M. M. Teracy, T. W. Ebbesen and J. M. Gibson: Nature 381 (1996) 678.

11 E. E. Wong, P. E. Sheehan and C. M. Lieber: Science 277 (1997) 1971.

12 S. Akita, H. Nishijima, Y. Nakayama, F. Tokumasu and K. Takeyasu: J. Phys. D 32 (1999) 1044.

13 S. Akita, S. Kamo and Y. Nakayama: Jpn. J. Appl. Phys. 41 (2002) L487.

14 K. Yamamoto, S. Akita and Y. Nakayama: J. Phys. D 31 (1998) L34.

15 B. Ilic and H. G. Craighed: J. Appl. Phys. 95 (2004) 7.

16 M. Ishida, J. Fujita, T. Ichihashi, Y. Ochiai, T. Kaito and S. Matsui: J. Vac. Sci. Technol. B 21 (2003) 2728.

17 N. V. Lavrik and P. G. Datskos: Appl. Phys. Lett. 82 (2003) 2697. 